\title{
Model systems to study plague pathogenesis and develop new therapeutics
}

\author{
Matthew B. Lawrenz* \\ Center for Predictive Medicine for Biodefense and Emerging Infectious Diseases, Department of Microbiology and Immunology, University of Louisville School of \\ Medicine, Louisville, KY, USA
}

\section{Edited by:}

Kenneth Fields, University of Miami, USA

\section{Reviewed by:}

Peter Dube, University of Texas Health

Science Center, USA

Gregory Plano, University of Miami,

USA

\section{*Correspondence:}

Matthew B. Lawrenz, Center for

Predictive Medicine, University of

Louisville, 505 S. Hancock St.

Louisville, KY 40202, USA.

e-mail:matt.lawrenz@louisville.edu
The Gram negative bacterium Yersinia pestis can infect humans by multiple routes to cause plague. Three plague pandemics have occurred and $Y$. pestis has been linked to biowarfare in the past. The continued risk of plague as a bioweapon has prompted increased research to understand $Y$. pestis pathogenesis and develop new plague therapeutics. Several in vivo models have been developed for this research and are reviewed here.

Keywords: Yersinia pestis, animal models, plague vaccines

\section{INTRODUCTION}

Plague is caused by the Gram negative pathogen Y. pestis. The bacterium exists in nature in a zoonotic cycle between mammalian hosts and the flea transmission vector (Perry and Fetherston, 1997; Ligon, 2006). Contact with infected fleas or animals can result in the transmission of $Y$. pestis to humans. In humans, plague infection can manifest in one of three forms based on transmission route and tissues infected. Bubonic plague is a result of flea transmission and is characterized by the development of swollen, infected lymph nodes called buboes. The bacteria will eventually enter the blood stream to cause septicemic plague and disseminate to other tissues. Colonization of the lungs results in secondary pneumonic plague and the possibility of person to person transmission through aerosol droplets (primary pneumonic plague). Plague infection rapidly progresses and is associated with a high mortality rate in untreated individuals (70-100\%). Successful treatment of infection is greatly increased with early detection.

Three plague pandemics have occurred, resulting in the death of over a third of the population of Europe and impacting the development of Western societies (Perry and Fetherston, 1997; Ligon, 2006). While plague has been considered a historic disease, concerns about the use of $Y$. pestis as a biological weapon and isolation of antibiotic resistant $Y$. pestis strains from nature have increased efforts to understand plague pathogenesis and develop novel therapeutics (Galimand et al., 1997; Inglesby et al., 2000). Researchers are equipped with a variety of tools to dissect the virulence mechanisms of $Y$. pestis and develop new approaches to combat the potential use of plague as a bioweapon. Here, we will discuss models of plague infection, their potential for use in defining plague pathogenesis, and their importance in translational research.

\section{HUMAN PLAGUE}

Humans are considered accidental hosts for Yersinia pestis and are extremely susceptible to infection. Human plague can present in infected patients in three forms: bubonic, septicemic, and pneu- monic. Bubonic plague, the most common form, arises after transmission of Y. pestis from an infected flea. The incubation period for bubonic plague is 2-8 days after exposure. During this incubation period, Y. pestis disseminates from the bite site to the regional lymph node. There the bacteria evade immune clearance and proliferate to high numbers. Patients initially present with flu-like symptoms, highlighted by the sudden onset of fever, chills, lethargy, and headache (Butler, 1983; Dennis, 2005; Adamovicz and Worsham, 2006). As the bacteria continue to proliferate, patients develop extremely painful swollen lymph nodes called buboes. Eventually these tissues will contain enormous numbers of extracellular bacteria. Typically patients present with a single bubo at the draining lymph node of the bite site. Infected lymph nodes become severely damaged, and pathology is characterized by hemorrhage and necrosis (Flexner, 1901). Without treatment, bubonic plague mortality rates approach $60 \%$. However, bubonic plague responds well to antibiotic treatment, and mortality rates with proper treatment have dropped to below 5\% (Dennis, 2005).

Bubonic plague can progress to septicemic plague if bacteria enter the blood stream. Septicemic plague is characterized by high bacteremia and is accompanied by a dangerous endotoxemia. In rare cases, $Y$. pestis can directly infect the blood and cause septicemic plague without presenting with symptoms of bubonic plague (called primary septicemic plague). Septicemic patients often have fever, severe headache, and lethargy but may also present with gatrointestinal symptoms (nausea, vomiting, diarrhea, and abdominal pain). Without the clinical development of buboes, septicemic patients are often not diagnosed with plague until Y. pestis is identified in blood smears. By this time the prognosis for infected patients is poor, and mortality rates are high even with antibiotic treatment (Butler, 1983).

In a small percentage of bubonic patients, $Y$. pestis can spread hematogenously to other tissues, including the lungs. Lung colonization can lead to the development of secondary pneumonic plague 
and the possibility of person to person transmission. Inhalation of aerosols containing $Y$. pestis can result in primary pneumonic plague in naive individuals. Primary pneumonic plague has a short incubation period of 1-2 days, followed by sudden onset of symptoms (fever, headache, chest pain, cough) and rapid progression of infection. Patients can present with localized bronchopneumonia or segmental to confluent consolidation of the lungs. Primary pneumonic infection is an extremely acute disease. Death can occur in as little as 3 days post-exposure. Furthermore, for antibiotic treatment to be effective, therapy needs to administered within $20 \mathrm{~h}$ of onset of illness (McCrumb et al., 1953; Butler, 1983). While pneumonic infection is a rare occurrence under natural situations, because of its ability for aerosol transmission and the acute nature of the infection, Y. pestis is also a potential bioweapon (Inglesby et al., 2000; Dennis, 2005). Prophylactic antibiotic treatment after a possible exposure can protect individuals from developing pneumonic plague, but new therapeutics and vaccines are needed to ensure protection in the event of a biological attack with weaponized Y. pestis.

Currently, no FDA licensed plague vaccines are available in the United States. However, both killed and live-attenuated vaccine candidates have been used in the past. Haffkine first described the use of a killed vaccine in 1879. Individuals were vaccinated with a heat-killed culture of fully virulent $Y$. pestis. Side effects associated with the vaccine included high incidence of localized pain, lymphadenopathy, fever, headache, and weakness. A small number of immunized individuals still contracted plague, suggesting that the vaccine was not fully protective (Meyer et al., 1974a). A formalinkilled vaccine called Plague Vaccine, USP was developed in the 1930 s and administered to military personnel. Vaccination with Plague Vaccine, USP elicited antibody responses in humans and is believed to have protected personnel against plague infection during the Vietnam War (Meyer, 1970). However, adverse reactions similar to the heat-killed vaccine were also reported in Plague Vaccine, USP vaccinated individuals. Furthermore, evidence from vaccinated rodents, non-human primates (NHPs), and humans suggests that the formalin-killed vaccine did not provide complete protection against pneumonic infection (Smiley, 2008a). The Y. pestis E.V. strain (and its derivative EV76) is a pigment-negative attenuated strain that has been used as a live vaccine in millions of people. Vaccination with this strain appears to provide protection against both bubonic and pneumonic plague. However, as a live bacterial vaccine, safety concerns are associated with vaccination and stem from a high frequency of debilitating side effects in humans and reports of primates dying as a result of vaccination (Meyer et al., 1974a; Smiley, 2008a).

More recently a subunit vaccine containing the F1 and LcrV antigens of $Y$. pestis has entered into clinical trials. Phase 1 trials to examine safety and immunogenicity of the F1 and LcrV subunits demonstrated that vaccination resulted primarily in a $\mathrm{TH} 2$ response of varying degrees (Williamson et al., 2005). Sera from vaccinated individuals passively protected mice from $Y$. pestis infection, indicating that protective immunity was achieved. However, antibody titers did not correlate with degree of protection. Studies to further examine the efficacy of this vaccine and others is difficult due to the low number of human plague cases. Future studies will likely rely on the results of animal vaccination studies and development of protective correlates that can be tested in humans.

\section{MODELS TO STUDY YERSINIA PESTIS PATHOGENESIS AND DEVELOP NEW PLAGUE THERAPEUTICS}

With the growing concern about antibiotic resistant strains of $Y$. pestis (Galimand et al., 1997) and the potential risk of a plague bioweapon (Inglesby et al., 2000), development of plague vaccine candidates and new post-exposure treatments are becoming increasingly important. These objectives have driven research to better understand the interactions between $Y$. pestis and its hosts. Systems to model insect and human infection are essential for these studies. Animal models of plague have been used by researchers for over 100 years. These important studies have included multiple different species and have established the progression of plague pathology and the potential of vaccine candidates (Adamovicz and Worsham, 2006). More recently, researchers have further characterized specific models to provide powerful tools to dissect aspects of $Y$. pestis pathogenesis, including interactions between the bacterium and host cells, transmission from the insect vector, infection of the mammalian host, and host immune responses during infection. This review will highlight recent developments in several common models used for plague research to understand $Y$. pestis pathogenesis and develop new therapeutic and vaccine candidates.

\section{INVERTEBRATE MODELS TO STUDY TRANSMISSION FACTORS}

$Y$. pestis is the only enteric bacterium to use an insect vector as its primary mode of transmission. The acquisition of genetic elements referred to as transmission factors allows for successful transmission from insect to mammal and back to insect. Transmission factors appear to be distinct from virulence genes that are required to cause mammalian disease (Hinnebusch et al., 1996; Paskewitz, 1997). Y. pestis primarily uses fleas for transmission and infects at least 80 different species (Pollitzer, 1954; Hinnebusch, 2005). In 1914, Bacot and Martin first described the mechanism for Y. pestis flea transmission using the rat flea Xenopsylla cheopis, which is a very efficient species for transmission (Bacot and Martin, 1914; Hirst, 1923). Their studies were highlighted by the observation that $Y$. pestis forms bacterial aggregates that block the feeding apparatus (proventriculus) of the flea. This blockage inhibits successful feeding by the flea and results in regurgitation of Y. pestis-infected blood back into the bite site and transmission of the bacterium into the mammalian host.

As the flea is the natural transmission vector for $Y$. pestis, it is a logical system to study $Y$. pestis-insect interactions. Furthermore, because $X$. cheopis is highly efficient in transmitting $Y$. pestis to the mammalian host in the laboratory setting, it has been used as a model to identify Y. pestis transmission factors (Hinnebusch, 2005). For example, the Yersinia murine toxin (Ymt) was first described due to its toxicity to mice (Warren et al., 1955). However, the $y m t$ gene was subsequently shown to be dispensable during mouse infection, and ymt mutants are fully virulent (Drozdov et al., 1995; Hinnebusch et al., 1996). While Ymt is not a virulence factor in mammals, it appears to be an important transmission factor. Studies by Hinnebusch et al. with the flea model demonstrated that Ymt is required for colonization of the flea midgut. $y m t^{(-)}$mutants are rapidly cleared from the midgut before the bacteria block the proventriculus (Hinnebusch et al., 2002). Interestingly, transfer of ymt into Y. pseudotuberculosis significantly increased the ability of this enteric pathogen to survive in the flea midgut. These findings 
suggest that the acquisition of $y m t$ was an important step in the evolution of $Y$. pestis from Y. pseudotuberculosis to become a vectorborne pathogen (Hinnebusch et al., 2002).

Using $X$. cheopis, researchers have also demonstrated that biofilm production by $Y$. pestis is essential for proventriculus blockage and transmission (Hinnebusch et al., 1996; Jarrett et al., 2004a). Biofilm production has been attributed to a set of genes in the pigmentation ( $\mathrm{ggm}$ ) locus called the hemin storage ( $\mathrm{hms}$ ) locus. Hinnebusch and colleagues have demonstrated that unlike the $y m t$ mutant, hms mutants are capable of prolonged colonization of the flea midgut. However, using microscopy, the researchers showed that $h m s$ mutants are defective in colonizing the proventriculus and forming aggregates. As proventriculus blockage is required for efficient transmission, $h m s$ mutants are also defective in transmission from flea to mammal. Through use of the flea model, the researchers demonstrated that $Y$. pestis transmission can depend on factors that are not required for pathogenesis in the mammal.

While the flea is the natural insect vector for Y. pestis, the model poses some difficulties that may limit its widespread use in Y.pestis studies. These include a requirement for dedicated and specialized facilities to work with fleas, relatively slow growth of the insects, and a risk for $Y$. pestis transmission from flea to human. A second invertebrate model that has recently emerged for studying $Y$. pestis-host interactions is the nematode Caenorhabditis elegans (Darby et al., 2002, 2005; Joshua et al., 2003; Styer et al., 2005; Bartra et al., 2008). C. elegans is a simple, tractable model system that has been used to study pathogenesis of many microbes (see review by Darby, 2005). The nematode is easy to maintain and grows quickly. In addition, C. elegans grows at a similar temperature as $X$. cheopis $\left(20-22^{\circ} \mathrm{C}\right)$. This means that many of the $Y$. pestis temperature regulated genes that are expressed during flea infection will likely also be expressed during C. elegans infection. Therefore, C. elegans may provide a simple system to screen for colonization and transmission factors of $Y$. pestis prior to further work with $X$. cheopis.

As seen in the flea, Y. pestis forms biofilms during C. elegans infection (Darby et al., 2002; Joshua et al., 2003). Biofilm formation inhibits the ability of $C$. elegans to feed and decreases survival of the worms. Mutational analysis confirmed that the $h m s$ genes were required for both biofilm formation and C. elegans death. Additional studies have demonstrated that $C$. elegans can be used to identify new potential transmission factors. Using a genetic screen, Darby et al. (2005) demonstrated that the gmhA gene of Y. pestis is involved in biofilm formation in C. elegans and is required for blockage of the flea proventriculus. In addition, Styer et al. (2005) have demonstrated that $Y$. pestis can kill C. elegans through a biofilm-independent mechanism. The researchers showed that the Y.pestis KIM5 strain, which lacks the hms locus and the ability to make biofilms, colonizes the nematode intestinal tract and causes a lethal infection. They further demonstrated that the $\mathrm{pCD} 1$ and $\mathrm{pPCP} 1$ plasmids were not required for death, indicating that the genes on these plasmids do not contribute to nematode colonization or death. Using a mutagenesis approach, the researchers also identified six novel Y. pestis factors that contributed to C. elegans death. They further demonstrated that two of these mutants were attenuated in the mouse model. These findings suggest that in addition to a model to identify $Y$. pestis factors important for the insect portion of the Y. pestis lifecycle, C. elegans may also be used to identify new pathogenesis factors involved in mammalian disease.

\section{THE MACROPHAGE MODEL TO STUDY PLAGUE INTERACTIONS WITH HOST CELLS}

Cavanaugh and Randall first suggested that $Y$. pestis entering the mammalian host from infected fleas are not resistant to phagocytosis and can be phagocytized by either monocytes or polymorphonuclear cells (PMNs). In these studies, bacteria that are phagocytized by PMNs were readily killed, but bacteria phagocytized by monocytes survived, replicated, and emerged resistant to further phagocytosis by both cell types (Cavanaugh and Randall, 1959). These observations have been confirmed by electron microscopy of monocytes from plague infected Rhesus macaques and by flow cytometry of infected cells in the spleens of plague infected mice (Finegold, 1969; Lukaszewski et al., 2005). Furthermore, depletion of macrophages prior to $Y$. pestis infection resulted in a better outcome for the host (Ye et al., 2009). These findings have contributed to the hypothesis that $Y$. pestis is a facultative intracellular pathogen that invades macrophages early in the infection process to avoid clearance by PMNs, and later emerges from macrophages expressing virulence factors (Caf1, T3SS effectors, etc.) to inhibit subsequent phagocytosis. $Y$. pestis invasion of macrophages may also contribute to the delay in the host immune response seen during plague infection.

In vitro studies with macrophages have confirmed in vivo findings that $Y$. pestis is a facultative intracellular pathogen (Janssen and Surgalla, 1969; Straley and Harmon, 1984a,b; Charnetzky and Shuford, 1985; Pujol and Bliska, 2003). Upon phagocytosis, $Y$. pestis enters into a phagocytic vacuole called the Yersinia containing vacuole (YCV). YCVs acquire markers of late endosomes and lysosomes, but $Y$. pestis appears to inhibit their normal acidification, suggesting that it resides within macrophages in modified phagolysosomal compartments (Straley and Harmon, 1984b; Grabenstein et al., 2006; Pujol et al., 2009). The bacteria remain in these spacious vacuoles and eventually begin to replicate. The mechanisms used by Y. pestis, and the host factors targeted by the bacterium to alter the normal phagocytic pathway, are not well understood. However, Straley and Harmon have demonstrated that virulence factors known to be important for mammalian infection, including factors on the $\mathrm{pCD} 1$ virulence plasmid, are not required for survival in macrophages (Straley and Harmon, 1984a). These findings suggest that additional factors are important for macrophage infection.

Several studies have demonstrated that the in vitro macrophage model can be used to identify intracellular pathogenicity factors. Oyston et al. (2000) demonstrated that survival of Y. pestis in J774A.1 macrophage-like cells is dependent on the two component regulator PhoP/Q. Graberstein et al. (2006) further demonstrated that phoP mutants failed to develop spacious vacuoles in infected J774A.1 cells and that three genes regulated by PhoP (ugd,pmrK, and $m g t C$ ) contribute to $Y$. pestis survival in these cells. Furthermore, $Y$. pestis phoP mutants were also attenuated in mouse infection, supporting the hypothesis that macrophage survival may be important for Y. pestis virulence (Oyston et al., 2000). Unlike $p h o P / Q$, the rip operon appears to specifically contribute to survival of $Y$. pestis in activated macrophages (Pujol et al., 2005). However, the contribution of the rip operon to mammalian virulence has not yet been determined. Together, these findings demonstrate that the macrophage model can be used to identify pathogenesis factors contributing to intracellular aspects of infection. Furthermore, as 
we gain more understanding of the intracellular lifecycle of $Y$.pestis, the macrophage model will be important in developing therapeutic strategies targeted at intracellular $Y$. pestis.

\section{MAMMALIAN MODELS TO STUDY PLAGUE PATHOGENESIS}

Animal models ranging from rodents to NHPs have been used to study plague pathogenesis over the years. By far rodent models are the predominate choice of plague researchers. This choice is based on many factors, including cost, small size of the rodent models, and availability of facilities to perform containment work. However, vaccine and therapeutic studies require testing in NHPs prior to FDA approval. While all of the models discussed here are susceptible to plague infection, each have caveats that could influence a researcher's selection of a plague model.

\section{The mouse model}

The mouse is currently the most widely used animal model for plague research. The mouse is highly susceptible to plague infection and appears to be equally susceptible to different strains of $Y$. pestis (Butler, 1983; Adamovicz and Worsham, 2006). Furthermore, the mouse model is susceptible to pneumonic and intravenous infection (but not bubonic infection) by strains of $Y$. pestis lacking the $p g m$ locus (Burrows and Jackson, 1956; Une and Brubaker, 1984). $\mathrm{ggm}^{(-)}$strains can be handled at BSL-2 and are exempt from Select Agent status (http://www.selectagents.gov/Exclusions. html). Consequently, the mouse model can be used with $\mathrm{pgm}^{(-)}$ strains by researchers who lack BSL-3 laboratories. However, while $\mathrm{pgm}^{(-)}$strains have been a useful tool to study plague, researchers need to take into consideration that $\mathrm{pgm}^{(-)}$strains are attenuated. Therefore, it is prudent that findings with $\mathrm{pgm}^{(-)}$strains, especially in the context of the host, be confirmed with fully virulent $Y$. pestis to ensure that the host does not react differently to the attenuated strain.

Recently, the kinetics of disease progression in the mouse model have been well characterized using the CO92 strain of Y. pestis (Lathem et al., 2005; Cathelyn et al., 2006; Agar et al., 2008). To model bubonic infection, mice can be infected via subcutaneous (s.c.) or intradermal injection. Disease progression via both routes is similar to transmission from infected fleas (Jarrett et al., 2004b). The $\mathrm{LD}_{50}$ during s.c. infection in this model is less than 10 colony forming units (cfu) (Cathelyn et al., 2006). After inoculation with $\sim 10^{2} \mathrm{cfu}, Y$. pestis can be detected in the draining lymph nodes as early as $24 \mathrm{~h}$ post-infection (M.B. Lawrenz and V.L. Miller, unpublished data). The bacteria continue to replicate and reach high concentrations in these tissues, similar to human plague. However, lymphadenopathy may not be obvious until later stages of infection. Systemic infection begins 12-24 h after lymph node colonization and is evident by isolation of bacteria from the spleen at $48 \mathrm{~h}$ postinfection. Lung colonization (secondary pneumonic plague) via systemic spread occurs after spleen colonization but is seen prior to the animals succumbing to infection. Death typically occurs between 3 and 5 days post-infection (Cathelyn et al., 2006).

Pneumonic infection of the mouse model can be achieved by intranasal or aerosol inoculation and results in an infection similar to human pneumonic plague (Lathem et al., 2005; Agar et al., 2008). The $\mathrm{LD}_{50}$ for intranasal infection with $Y$. pestis CO92 appears to be approximately 10 -fold less than for aerosol inoculation $(\sim 250 \mathrm{cfu}$ vs. $2100 \mathrm{cfu}$, respectively), although only approximately $10 \%$ of the inoculum reaches the lungs via intranasal inoculation (Meyer, 1950; Lathem et al., 2005). Bacterial numbers in the lungs steadily increase between 1 and $72 \mathrm{~h}$ post-infection regardless of inoculation method. Lung pathology appears mild at $24 \mathrm{~h}$ but dramatically worsens over the course of infection, resulting in large areas of consolidation and inflammation by $72 \mathrm{~h}$. As seen in primary human pneumonic plague, bacteria disseminate from the lungs of infected mice to colonize the spleen by about $36 \mathrm{~h}$ post-infection. Interestingly, despite spleen colonization, Agar et al. did not detect bacteria in the blood until $72 \mathrm{~h}$ after aerosol inoculation (about $24 \mathrm{~h}$ later than from intranasal infection (Sha et al., 2008)) when concentrations were greater than $10^{4} \mathrm{cfu} / \mathrm{ml}$ (Agar et al., 2008). By both aerosol and intranasal infection, mice typically succumb to pneumonic disease 72-96 h after exposure (Lathem et al., 2005; Agar et al., 2008).

The mouse pneumonic plague model demonstrated that a biphasic immune response occurs during plague infection (Lathem et al., 2005; Bubeck et al., 2007; Agar et al., 2008). During the first $48 \mathrm{~h}$ of infection, $Y$. pestis steadily grows in the lungs of infected mice in the apparent absence of recognition by the host immune system. Concurrent with systemic spread of the infection, a sudden and strong inflammatory response is mounted by the host. This response includes induction of proinflammatory cytokines and chemokines and infiltration of PMNs into the lungs. Despite this massive response, Y. pestis appears to continue to grow unchecked. The combination of lung damage and an uncontrolled immune response results in death of the animals. This biphasic or delayed immune response differs from other Gram negative pneumonias. In the mouse model for Klebsiella pneumoniae, for example, mice mount a strong proinflammatory response within $24 \mathrm{~h}$ of intranasal infection, which includes induction of proinflammatory cytokines and chemokines and recruitment of PMNs into the lungs (Lawlor et al., 2006; Bubeck et al., 2007). These findings support the hypothesis that $Y$. pestis actively modulates the immune response through effectors (Yops and LcrV) secreted by the type III secretion system encoded on the pCD1 virulence plasmid (see Cornelis and WolfWatz, 1997; Viboud and Bliska, 2005 for reviews).

The mouse model has also been widely used to study interactions between $Y$. pestis and the mammalian immune system (for detailed reviews see Li and Yang, 2008; Smiley, 2008b). The availability of knockout and transgenic mice continues to make this an attractive model for immune system interaction studies. For example, from in vitro models and studies with enteric Yersinia, it has been hypothesized that induction of IL-10 is important for Y. pestis to establish infection (Sing et al., 2002a,b). Despite this evidence, several studies of cytokine expression during plague infection have not been able to demonstrate a significant elevation in IL-10 levels in infected animals, suggesting that IL-10 does not play a role in plague infection (Lathem et al., 2005; Bubeck et al., 2007; Agar et al., 2008). Recently, DePaolo and colleagues used IL-10 $0^{(-/)}$knockout mice to test the hypothesis that IL-10 is involved in Y. pestis infection. The researchers demonstrated that IL- $10^{(--)}$knockout mice displayed a significantly different immune response against $Y$. pestis CO92 and were protected from plague infection compared to wild type mice, supporting the hypothesis that $Y$. pestis induces IL-10 to establish infection (DePaolo et al., 2008). 
The mouse model has also served as a tool to begin to understand host determinants that may contribute to resistance to $Y$. pestis infection (Congleton et al., 2006; Turner et al., 2008, 2009; Blanchet et al., 2010). Recently, Blanchet et al. identified that the SEG mouse, an inbred Mus spretus line, is significantly more resistant to infection by fully virulent $Y$. pestis compared to other more commonly used Mus musculus inbred lines such as C57BL/6. The subcutaneous $\mathrm{LD}_{50}$ in female SEG mice appears to be greater than $10^{7}$ bacteria compared to less than 10 for C57BL/6. The researchers identified three independent quantitative trait loci (QTL) that contribute to resistance to $Y$. pestis infection in an additive fashion (Blanchet et al., 2010). Although further analysis of these loci is needed to identify the genes responsible for increased resistance to plague infection, these studies may provide insight into possible differences in plague susceptibility in the human population.

The advantages of the mouse model to study pathogenesis also apply to its use in testing potential plague therapeutics. Numerous potential vaccines, including subunit, live-attenuated, killed, and DNA vaccine candidates, have been screened for efficacy in the mouse model (see Smiley, 2008a for review of vaccine candidates). In addition, novel therapeutics including monoclonal antibodies, new antimicrobial compounds, and new adjuvants to augment vaccine efficacy have also been studied in the mouse plague model (Uddowla et al., 2007; Amemiya et al., 2009; Eisele and Anderson, 2009). However, it is important to consider that the differences between mouse and human anatomy and physiology may impact interpretations of therapeutic studies. For example, the respiratory tracts of mice and humans are dramatically different. Compared to humans, mice have increased surface area in their nasal passages, have only a single lobe in their left lung, lack bronchioles, are unable to cough in response to mechanical stimulation, and exhibit different immune potentials in the lungs (Irvin and Bates, 2003; Mizgerd and Skerrett, 2008). Furthermore, the immune system of mice is different than that of humans (Mestas and Hughes, 2004). Therefore, results from rodent models, especially in the case of therapeutic studies, need to be confirmed in models more closely related to humans, such as NHPs (see below), prior to being directly applied to human disease.

\section{The guinea pig model}

The guinea pig has been used as a plague model since the early $1900 \mathrm{~s}$ and is still used in vaccine studies today (Jones et al., 2003; Qi et al., 2010). Like the other small animal models, the guinea pig is highly susceptible to $Y$. pestis infection (subcutaneous $\mathrm{LD}_{50}<10$, aerosol $\mathrm{LD}_{50} \sim 40,000$ ) (Welkos et al., 1995; Titball and Williamson, 2001). Subcutaneous infection of the guinea pig results in the development of papules at the infection site and lymphadenopathy in the draining lymph nodes. As in human plague, guinea pigs eventually develop septicemic infection and succumb to disease within 2 weeks (Pollitzer, 1954). It is important to note that the pneumonic guinea pig model can differ dramatically from the mouse and NHP models. Aerosol infections that initiate a primary pneumonia in mice or NHPs may not cause primary pneumonia in the guinea pig. $Y$. pestis introduced into guinea pigs by aerosol inoculation tends to initially colonize the cervical lymph nodes, indicating that infection originates from the upper respiratory tract. Infection of the lungs can occur, but it is delayed and appears to be a secondary pneumonia resulting from dissemination through the blood stream (Strong and Teague, 1912a,b; Meyer, 1961). Evidence suggests that aerosol particle size determines whether primary pneumonia will develop. Compared to other models, it appears smaller particle sizes are required to deliver $Y$. pestis into the lungs of guinea pigs (Druett et al., 1956). Furthermore, intranasal inoculation does not appear as reliable in the guinea pig as reported for the mouse model and may not be a viable route for infection (Meyer, 1961).

The guinea pig can also show striking differences in susceptibility to different $Y$. pestis strains compared to other models. For example, the guinea pig model is highly resistant to auxotrophic strains that retain virulence in mice and NHPs (Burrows, 1960, 1963; Burrows and Gillett, 1971; Meyer et al., 1974b; Oyston et al., 1996). It has been suggested that physiological differences in free amino acids between species may be responsible for the differences seen in susceptibility. The guinea pig is also resistant to infection by Y. pestis strains lacking the F1 capsule that are virulent in the mouse model (Burrows and Bacon, 1958; Welkos et al., 1995). Therefore, it may be prudent to test any attenuated mutants or potential live vaccine candidates identified in the guinea pig model in other small animal models prior to progressing to primate studies.

\section{The rat model}

The rat has been intimately linked to the plague throughout history and is often associated with urban outbreaks of human plague. Like the mouse, the genetics and immunology of the rat have been well characterized, making it a useful tool for biomedical research. While susceptible to $Y$. pestis infection, until recently the rat has not been widely used in pathogenesis studies because of perceived variability in disease progression during infection. Chen and Meyer (1974) reported that both laboratory and wild rats appear to have an increased resistance to plague infection compared to other rodent models. These studies indicated that the lethal dose could be as much as 1,000-fold higher for a subcutaneous infection compared to the mouse model. More recent work with the inbred Brown Norway strain of Rattus norvegicus suggests that the rat can be a reproducible model to study human plague. Disease manifestation in this rat strain closely recapitulates human infection and may be especially useful in studying interactions in the draining lymph nodes during bubonic infection. Sebbane et al. extensively characterized the Brown Norway model for bubonic plague infection. These studies demonstrated that Brown Norway rats are relatively susceptible to plague infection $(\sim 250$ bacteria needed to cause bubonic infection) (Sebbane et al., 2005). As seen in human bubonic plague, the proximal lymph nodes were the first tissues to be infected in the rat, followed later by the blood, spleen, and lastly, the distal lymph nodes. The draining lymph node was rapidly colonized by $Y$. pestis (as early as 6 h post-infection), and extracellular bacteria were visible by $24 \mathrm{~h}$ post-infection. As the infection progressed, bacterial numbers dramatically increased, and the researchers observed lymphadenopathy, hemorrhage, necrosis, and other pathologies characteristic of human buboes. Eventually, bacteria spread to the blood (bacteremia) and disseminated through the blood to the spleen (as seen in septicemic plague). The authors also reported an apparent delayed immune response in the lymph nodes, which was ineffective in controlling infection, similar to what has been seen in mice and NHPs. 
More recently, researchers have further characterized the Brown Norway model during pneumonic infection (Agar et al., 2009; Anderson et al., 2009). Using intranasal inoculation, Anderson et al. (2009) demonstrated that the $\mathrm{LD}_{50}$ for Y. pestis CO92 in the rat was similar to that reported for mice $(\sim 200 \mathrm{cfu})$. Independent experiments by Agar et al. (2009) confirmed this intranasal $\mathrm{LD}_{50}$ and further demonstrated that the $\mathrm{LD}_{50}$ for aerosol transmission was also similar to mice $\left(\sim 1.6 \times 10^{3} \mathrm{cfu}\right)$. During intranasal infection, rats appeared healthy until $36 \mathrm{~h}$ post-infection, when they began to demonstrate a hunched posture. From this point the animal's condition quickly worsened, with an approximate mean day to death between 3 and 4 days postinfection. The authors reported that the animals did not appear to lose weight over the course of the infection (Anderson et al., 2009). One striking difference in rats infected via aerosol was an apparent earlier development of lung inflammation. Acute inflammation was observed by $24 \mathrm{~h}$ in aerosol infected animals, compared to inflammation occurring after $36 \mathrm{~h}$ in the intranasal rat and pneumonic mouse models (Lathem et al., 2005; Bubeck et al., 2007; Agar et al., 2008). The rat pneumonic model also differed in the kinetics of bacterial growth in the lungs. Unlike the mouse model, which demonstrates a steady increase in bacterial numbers in the lungs from 12 to $72 \mathrm{~h}$ (Lathem et al., 2005), Y. pestis numbers did not appear to increase in the lungs of infected rats between 12 and $48 \mathrm{~h}$, regardless of inoculation method (Agar et al., 2009; Anderson et al., 2009). During this time the bacteria disseminated into the blood and to other tissues. At $48 \mathrm{~h}$, bacterial numbers began to increase in the lungs. It is unclear whether this increase was due to outgrowth of $Y$. pestis in the lungs or arrival of systemic organisms to the lungs through recirculation. As observed in the mouse (Lathem et al., 2005; Bubeck et al., 2007; Agar et al., 2008), the rat pneumonic model demonstrated that $Y$. pestis alters the immune response of its host to delay the expression of proinflammatory cytokines and chemokines (Agar et al., 2009). Furthermore, Agar et al. (2009) were also able to show rat to rat transmission ( $-33 \%$ transmission rate), which has not been shown in mice. This finding suggests that the rat may be used to model person to person transmission of primary pneumonic plague.

The similarity in lymph node disease between humans and the rat suggests that the rat may be a particularly relevant model to examine pathogenesis in these tissues. Using the rat lymph node model, Sebbane et al. (2006) examined the Y. pestis gene expression profile in infected lymph nodes. This work demonstrated that in response to a strong host iNOS response in the lymph nodes, $Y$. pestis genes that protect the bacteria from reactive nitrogen species were upregulated. The work further demonstrated that inactivation of one of these upregulated genes, $h m p$, resulted in an increase in the $\mathrm{LD}_{50}$ of $Y$. pestis in the rat. Future studies to analyze host $Y$. pestis interactions in the lymph node could benefit from the rat model.

\section{NON-HUMAN PRIMATE MODELS OF PLAGUE INFECTION}

Non-human primates research is expensive and requires specialized expertise and facilities, which imposes limitations on its widespread use to study Y. pestis pathogenesis (Patterson and Carrion, 2005). Regardless, the close relationship of humans to NHPs make these important models to study plague and have provided useful insight into $Y$. pestis disease progression. Future studies with NHPs will be important in the successful development of plague vaccines and therapeutics. Three of the more widely used species to study plague are described here.

\section{Rhesus macaques}

Progression of pneumonic plague in Rhesus macaques appears to be very similar to human infection (Speck and Wolochow, 1957; Finegold et al., 1968). As in humans, the disease has a short incubation period and animals usually show the first sign of infection (fever) within 2 days after exposure. The mean day to death for infected Rhesus macaques is approximately 6 days, and animals developed all stages of pneumonia during the course of infection. Although disease progression appears similar to humans, Rhesus monkeys are significantly more resistant to plague infection (Ehrenkranz and Meyer, 1955; Speck and Wolochow, 1957). The $\mathrm{LD}_{50}$ for $Y$. pestis in the Rhesus model can be as much as 10 -fold higher for pneumonic infection and several thousand-fold higher for bubonic infection than other NHP models or the estimated infectious doses for humans. Furthermore, Rhesus macaques have demonstrated a tendency to develop a form of plague referred to as chronic pneumonic plague, which is very rarely seen in humans (Ransom and Krueger, 1954). These differences in the Rhesus model should be considered when using this model to evaluate pathogenesis of plague or new plague therapeutics.

\section{African green monkeys}

Unlike the Rhesus model, the African green monkey is highly susceptible to plague infection. The $\mathrm{LD}_{50}$ of $Y$. pestis appears to be slightly lower than the predicted doses for humans, with a $\mathrm{LD}_{50} \sim 300 \mathrm{cfu}$ by aerosol transmission and $\sim 5-50 \mathrm{cfu}$ by intradermal inoculation (Chen et al., 1977; Adamovicz and Worsham, 2006). Despite this increased susceptibility, disease manifestations and pathology in aerosol infected African green monkeys are similar to human pneumonic plague patients (Layton et al., 2010). Bacteria grow to high numbers in the lungs, resulting in severe pneumonia, and disseminate to the blood in as little as 2 days post-infection. Monkeys develop fever at various time points post-infection, but in most cases fever was not observed until after bacteremia and approximately $24 \mathrm{~h}$ prior to succumbing to infection. The mean time to death for aerosol infected African green monkeys is approximately 4 days post-exposure (Layton et al., 2010).

This model has shown some variability in susceptibility to the attenuated EV76 vaccine strain. This variability may be due to its increased susceptibility to $Y$. pestis as a species; however, some evidence suggests that variability could be attributed to the origin of the subspecies used in the studies (Hallett et al., 1973). African green monkeys have also demonstrated a high degree of variability in vaccine studies (Hallett et al., 1973; Chen et al., 1977; Pitt, 2004). Both subunit and live vaccine candidates that have shown protection in other animal models show only partial protection in this model. It has been difficult to interpret this data, especially in the context of its implications for human response to vaccination, but the African green monkey may be a more stringent model than other NHPs for testing vaccine efficacy (Pitt, 2004). Additionally, 
as these monkeys are outbred populations (unlike most rodent models), variability in responses to vaccination may be a result of this heterogeneity and a closer representation of the efficacy that will be seen in humans.

\section{Cynomolgus macaques}

The Cynomolgus macaque has a history of use as a model for plague vaccine and pathogenesis studies and has been suggested as a strong candidate model for continued plague vaccine research (Adamovicz and Worsham, 2006). Significant effort has been made recently to better characterize primary pneumonic plague in this model to improve future vaccine and therapeutic studies (Adamovicz and Worsham, 2006; Van Andel et al., 2008; Koster et al., 2010). These reports demonstrated that Cynomolgus macaques are highly susceptible to aerosolized plague $\left(\mathrm{LD}_{50}\right.$ $\sim 300 \mathrm{cfu}$ ), and infected animals develop clinical signs similar to human pneumonic plague. Most animals presented with fever as the earliest clinical symptom. Other clinical signs of infection were delayed for an additional $24 \mathrm{~h}$, at which time the macaques showed signs of lethargy and anorexia but not respiratory distress or coughing (despite histologic pathology demonstrating lung damage), which are commonly seen in humans. Bacteria eventually disseminated from the lungs to the spleen and liver via the blood. Using telemetry to detect fever and sensitive quantitative PCR techniques to detect $Y$. pestis in macaque blood, Koster et al. (2010) have suggested that the development of fever may be an indication of bacteremia and systemic disease. Using Cynomolgus macaques, Meyer et al. (1974a) have demonstrated that killed

\section{REFERENCES}

Adamovicz,J.J., and Worsham, P.L. (2006). "Plague," in Biodefense: Research Methodology and Animal Models, ed. J. R. Swearengen (Boca Raton: Taylor and Francis), 107-136.

Agar, S. L., Sha, J., Foltz, S. M., Erova, T. E., Walberg, K. G., Baze, W. B., Suarez, G., Peterson, J. W., and Chopra, A. K. (2009). Characterization of the rat pneumonic plague model: infection kinetics following aerosolization of Yersinia pestis CO92. Microbes Infect. $11,205-214$.

Agar, S. L., Sha, J., Foltz, S. M., Erova, T. E., Walberg, K. G., Parham, T. E., Baze, W. B., Suarez, G., Peterson, J. W., and Chopra,A.K. (2008). Characterization of a mouse model of plague after aerosolization of Yersinia pestis CO92. Microbiology 154, 1939-1948.

Amemiya, K., Meyers, J. L., Rogers, T. E., Fast, R. L., Bassett, A. D., Worsham, P. L., Powell, B. S., Norris, S. L., Krieg, A. M., and Adamovicz, J. J. (2009). $\mathrm{CpG}$ oligodeoxynucleotides augment the murine immune response to the Yersinia pestis F1-V vaccine in bubonic and pneumonic models of plague. Vaccine 27, 2220-2229.

Anderson, D. M., Ciletti, N. A., LeeLewis, H., Elli, D., Segal, J., DeBord, K. L., Overheim, K. A., Tretiakova, M.,
Brubaker, R. R., and Schneewind, O. (2009). Pneumonic plague pathogenesis and immunity in Brown Norway rats. Am. J. Pathol. 174, 910-921.

Bacot, A. W., and Martin, C. J. (1914). Observations on the mechanism of the transmission of plague by fleas. $J$. Hygiene Plague Suppl. 4, 774-776.

Bartra, S. S., Styer, K. L., O’Bryant, D. M., Nilles, M. L., Hinnebusch, B.J., Aballay, A., and Plano, G. V. (2008). Resistance of Yersinia pestis to complementdependent killing is mediated by the Ail outer membrane protein. Infect. Immun. 76, 612-622.

Blanchet, C., Jaubert, J., Carniel, E., Fayolle, C., Milon, G., Szatanik, M., Panthier, J. J., and Montagutelli, X. (2010). Mus spretus SEG/Pas mice resist virulent Yersinia pestis, under multigenic control. Genes Immun. doi: 10.1038/gene.2010.45.

Bubeck, S. S., Cantwell, A. M., and Dube, P. H. (2007). Delayed inflammatory response to primary pneumonic plague occurs in both outbred and inbred mice. Infect. Immun. 75, 697-705.

Burrows, T.W.(1960). Biochemical properties of virulent and avirulent strains of bacteria: Salmonella typhosa and Pasteurella pestis. Ann. N. Y. Acad. Sci. 88, 1125-1135.

vaccines are protective against bubonic plague, but do not protect against aerosol infection. These findings suggest that killed vaccines may not be effective against human pneumonic plague (Meyer, 1970; Meyer et al., 1974a).

Regardless of the NHP model used in vaccine studies, understanding correlates of protection and their value in predicting success in humans is an important challenge. In several studies, antibody titer has been shown to directly correlate with successful protection. However, specific examples have demonstrated that antibody titer may not guarantee successful vaccination and cellular responses should be also considered (see Smiley, 2008a for review of plague vaccine challenges). Therefore, further characterization of the NHP plague models and the immune responses to vaccines in these models will be crucial to establishing a successful plague vaccine.

\section{SUMMARY}

While the models highlighted in this review may be used interchangeably, specific aspects of an individual model may provide unique insights into Y. pestis pathogenesis (e.g., identification of transmission factors vs. pathogenesis factors vs. treatment development). A comprehensive understanding of the interactions between plague and its hosts will likely require data gathered from multiple models. As new anti-plague prophylactic and therapeutic candidates are developed, further characterization of plague pathogenesis and immune responses in these models will be important to understand correlates of protection that can be applied toward predicting success in humans.

Burrows, T. W. (1963). Virulence of Pasteurella pestis and immunity to plague. Ergeb. Mikrobiol. Immunitatsforsch. Exp. Ther. 37, 59-113.

Burrows, T. W., and Bacon, G. A. (1958) The effects of loss of different virulence determinants on the virulence and immunogenicity of strains of Pasteurella pestis. Br. J. Exp. Pathol. 39, 278-291.

Burrows, T. W., and Gillett, W. A. (1971) Host specificity of Brazilian strains of Pasteurella pestis. Nature 229, 51-52.

Burrows, T. W., and Jackson, S. (1956) The virulence-enhancing effect of iron on nonpigmented mutants of virulent strains of Pasteurella pestis. Br. J. Exp. Pathol. 37, 577-583.

Butler, T. (1983). Plague and Other Yersinia Infections. New York: Plenum Medical Book Company.

Cathelyn, J. S., Crosby, S. D., Lathem, W W., Goldman, W. E., and Miller, V. L. (2006). RovA, a global regulator of Yersinia pestis, specifically required for bubonic plague. Proc. Natl. Acad. Sci. U.S.A. 103, 13514-13519.

Cavanaugh, D. C., and Randall, R. (1959). The role of multiplication of Pasteurella pestis in mononuclear phagocytes in the pathogenesis of flea-borne plague. J. Immunol. 83, 348-363.
Charnetzky, W. T., and Shuford, W. W. (1985). Survival and growth of Yersinia pestis within macrophages and an effect of the loss of the 47-megadalton plasmid on growth in macrophages. Infect. Immun. 47, 234-241.

Chen, T. H., Elberg, S. S., and Eisler, D. M. (1977). Immunity in plague: protection of the vervet (Cercopithecus aethips) against pneumonic plague by the oral administration of live attenuated Yersinia pestis. J. Infect. Dis. 135, 289-293.

Chen, T. H., and Meyer, K. F. (1974). Susceptibility and antibody response of Rattus species to experimental plague. J. Infect. Dis. 129(Suppl.), S62-S71.

Congleton, Y. H., Wulff, C. R., Kerschen, E. J., and Straley, S. C. (2006). Mice naturally resistant to Yersinia pestis $\{$ Delta\}pgm strains commonly used in pathogenicity studies. Infect. Immun. 74, 6501-6504.

Cornelis, G. R., and Wolf-Watz, H. (1997). The Yersinia Yop virulon: a bacterial system for subverting eukaryotic cells. Mol. Microbiol. 23, 861-867.

Darby, C. (2005). Interactions with microbial pathogens. WormBook 1-15.

Darby, C., Ananth, S. L., Tan, L., and Hinnebusch, B. J. (2005). Identification of gmhA, a Yersinia pestis gene 
required for flea blockage, by using a Caenorhabditis elegans biofilm system. Infect. Immun. 73, 7236-7242.

Darby, C., Hsu, J. W., Ghori, N., and Falkow, S. (2002). Caenorhabditis elegans: plague bacteria biofilm blocks food intake. Nature 417, 243-244.

Dennis, D. T. (2005). "Plague as a biological weapon," in Bioterrorism and Infectious Disease: A New Dilemma for the 21st Century, eds I. W. Fong and K. Alibek (New York: Springer), 37-70.

DePaolo, R. W., Tang, F., Kim, I., Han, M., Levin, N., Ciletti, N., Lin, A., Anderson, D., Schneewind, O., and Jabri, B. (2008). Toll-like receptor 6 drives differentiation of tolerogenic dendritic cells and contributes to LcrV-mediated plague pathogenesis. Cell Host Microbe 4, 350-361.

Drozdov, I. G., Anisimov, A. P., Samoilova, S. V., Yezhov, I. N., Yeremin, S. A., Karlyshev, A. V., Krasilnikova, V. M., and Kravchenko, V. I. (1995). Virulent non-capsulate Yersinia pestis variants constructed by insertion mutagenesis. J. Med. Microbiol. 42, 264-268.

Druett, H.A., Robinson, J.M., Henderson, D. W., Packman, L., and Peacock, S. (1956). Studies on respiratory infection. II. The influence of aerosol particle size on infection of the guinea-pig with Pasteurella pestis. J. Hyg. (Lond.) 54, 37-48.

Ehrenkranz, N. J., and Meyer, K. F. (1955). Studies on immunization against plague. VIII. Study of three immunizing preparations in protecting primates against pneumonic plague. J. Infect. Dis. 96, 138-144.

Eisele, N. A., and Anderson, D. M. (2009). Dual-function antibodies to Yersinia pestis LcrV required for pulmonary clearance of plague. Clin. Vaccine Immunol. 16, 1720-1727.

Finegold, M.J. (1969). Pneumonic plague in monkeys. An electron microscopic study. Am. J. Pathol. 54, 167-185.

Finegold, M. J., Petery, J. J., Berendt, R. F., and Adams, H. R. (1968). Studies on the pathogenesis of plague. Blood coagulation and tissue responses of Macaca mulatta following exposure to aerosols of Pasteurella pestis. Am. J. Pathol. 53, 99-114.

Flexner, S. (1901). The pathology of bubonic plague. Am. J. Med. Sci. 122, 396-416.

Galimand, M., Guiyoule, A., Gerbaud, G., Rasoamanana, B., Chanteau, S., Carniel, E., and Courvalin, P. (1997). Multidrug resistance in Yersinia pestis mediated by a transferable plasmid. $N$. Engl. J. Med. 337, 677-680.

Grabenstein, J. P., Fukuto, H. S., Palmer, L. E., and Bliska, J. B. (2006). Characterization of phagosome trafficking and identification of PhoP- regulated genes important for survival of Yersinia pestis in macrophages. Infect. Immun. 74, 3727-3741.

Hallett, A. F., Isaacson, M., and Meyer, K. F. (1973). Pathogenicity and immunogenic efficacy of a live attenuated plague vaccine in vervet monkeys. Infect. Immun. 8, 876-881.

Hinnebusch, B. J. (2005). The evolution of flea-borne transmission in Yersinia pestis. Curr. Issues Mol. Biol. 7, 197-212.

Hinnebusch, B. J., Perry, R. D., and Schwan, T. G. (1996). Role of the Yersinia pestis hemin storage (hms) locus in the transmission of plague by fleas. Science $273,367-370$.

Hinnebusch, B. J., Rudolph, A. E., Cherepanov, P., Dixon, J. E., Schwan, T. G., and Forsberg, A. (2002). Role of Yersinia murine toxin in survival of Yersinia pestis in the midgut of the flea vector. Science 296, 733-735.

Hirst, L. F. (1923). On the transmission of plague by fleas of the genus Xenopsylla. Indian J. Med. Res. 10, 789-820.

Inglesby, T. V., Dennis, D. T., Henderson, D. A., Bartlett, J. G., Ascher, M. S., Eitzen, E., Fine, A. D., Friedlander, A. M., Hauer, J., Koerner, J. F., Layton, M., McDade, J., Osterholm, M. T., O’Toole, T., Parker, G., Perl, T. M., Russell, P. K., Schoch-Spana, M., and Tonat, K. (2000). Plague as a biological weapon: medical and public health management. Working Group on Civilian Biodefense. JAMA 283, 2281-2290.

Irvin, C. G., and Bates, J. H. (2003). Measuring the lung function in the mouse: the challenge of size. Respir. Res. 4,4 .

Janssen, W. A., and Surgalla, M. J. (1969). Plague bacillus: survival within host phagocytes. Science 163, 950-952.

Jarrett, C. O., Deak, E., Isherwood, K. E., Oyston, P. C., Fischer, E. R., Whitney, A. R., Kobayashi, S. D., DeLeo, F. R., and Hinnebusch, B. J. (2004a). Transmission of Yersinia pestis from an infectious biofilm in the flea vector. J. Infect. Dis. 190, 783-792.

Jarrett, C. O., Sebbane, F., Adamovicz, J. J., Andrews, G. P., and Hinnebusch, B. J. (2004b). Flea-borne transmission model to evaluate vaccine efficacy against naturally acquired bubonic plague. Infect. Immun. 72, 2052-2056.

Jones, S. M., Griffin, K. F., Hodgson, I., and Williamson, E. D. (2003). Protective efficacy of a fully recombinant plague vaccine in the guinea pig. Vaccine 21, 3912-3918.

Joshua, G. W., Karlyshev, A. V., Smith, M. P., Isherwood, K. E., Titball, R. W., and Wren, B. W. (2003). A Caenorhabditis elegans model of Yersinia infection: biofilm formation on a biotic surface. Microbiology 149, 3221-3229.

Koster, F., Perlin, D. S., Park, S., Brasel, T., Gigliotti, A., Barr, E., Myers, L., Layton, R. C., Sherwood, R., and Lyons, C. R. (2010). Milestones in progression of primary pneumonic plague in cynomolgus macaques. Infect. Immun. 78, 2946-2955.

Lathem, W. W., Crosby, S. D., Miller V. L., and Goldman, W. E. (2005). Progression of primary pneumonic plague: a mouse model of infection pathology, and bacterial transcriptional activity. Proc. Natl. Acad. Sci. U.S.A. 102, 17786-17791.

Lawlor, M. S., Handley, S. A., and Miller, V. L. (2006). Comparison of the host responses to wild-type and cpsB mutant Klebsiella pneumoniae infections. Infect. Immun. 74, 5402-5407.

Layton, R. C., Brasel, T., Gigliotti, A., Barr E., Storch, S., Myers, L., Hobbs, C., and Koster, F. (2010). Primary pneumonic plague in the African Green monkey as a model for treatment efficacy evaluation. J. Med. Primatol. doi 10.1111/j.1600-0684.2010.00443.x

Li, B., and Yang, R. (2008). Interaction between Yersinia pestis and the host immune system. Infect. Immun. 76 , 1804-1811.

Ligon, B. L. (2006). Plague: a review of its history and potential as a biological weapon. Semin. Pediatr. Infect. Dis. 17, 161-170.

Lukaszewski, R. A., Kenny, D. J., Taylor, R. Rees, D. G., Hartley, M. G., and Oyston, P. C. (2005). Pathogenesis of Yersinia pestis infection in $\mathrm{BALB} / \mathrm{c}$ mice: effects on host macrophages and neutrophils. Infect. Immun. 73, 7142-7150.

McCrumb, F. R. Jr., Mercier, S., Robic, J., Bouillat, M., Smadel, J.E., Woodward, T. E., and Goodner, K. (1953). Chloramphenicol and terramycin in the treatment of pneumonic plague. Am. J. Med. Sci. 14, 284-293.

Mestas, J., and Hughes, C. C. (2004). Of mice and not men: differences between mouse and human immunology. $J$. Immunol. 172, 2731-2738.

Meyer, K. F. (1950). Immunity in plague: a critical consideration of some recent studies. J. Immunol. 64, 139-163.

Meyer, K. F. (1961). Pneumonic plague. Bacteriol. Rev. 25, 249-261.

Meyer, K. F. (1970). Effectiveness of live or killed plague vaccines in man. Bull. World Health Organ. 42, 653-666.

Meyer, K. F., Cavanaugh, D. C., Bartelloni, P. J., and Marshall, J. D. Jr. (1974a). Plague immunization. I. Past and present trends. J. Infect. Dis. 129(Suppl), S13-S18.

Meyer, K. F., Smith, G., Foster, L., Brookman, M., and Sung, M. (1974b) Live, attenuated Yersinia pestis vac- cine: virulent in nonhuman primates, harmless to guinea pigs. J. Infect. Dis. 129(Suppl.), S85-S112.

Mizgerd, J. P., and Skerrett, S. J. (2008). Animal models of human pneumonia. Am. J. Physiol. Lung Cell Mol. Physiol. 294, L387-L398.

Oyston, P. C., Dorrell, N., Williams, K., Li, S. R., Green, M., Titball, R. W., and Wren, B. W. (2000). The response regulator PhoP is important for survival under conditions of macrophage-induced stress and virulence in Yersinia pestis. Infect. Immun. 68, 3419-3425.

Oyston, P. C., Russell, P., Williamson, E. D., and Titball, R. W. (1996). An aroA mutant of Yersinia pestis is attenuated in guinea-pigs, but virulent in mice. Microbiology 142(Pt 7), 1847-1853.

Paskewitz, S.M. (1997). Transmission factors for insect-vectored microorganisms. Trends Microbiol. 5, 171-173.

Patterson, J. L., and Carrion, R. Jr. (2005) Demand for nonhuman primate resources in the age of biodefense. ILAR J. 46, 15-22.

Perry, R. D., and Fetherston, J. D. (1997). Yersinia pestis: etiologic agent of plague. Clin. Microbiol. Rev. 10, 35-66.

Pitt, L. (2004). "Nonhuman primates as a model for pneumonic plague," in Public Workshop on Animal Models and Correlates of Protection for Plague Vaccines, Gaithersburg, MD. Available at: http://www.fda.gov/downloads/ BiologicsBloodVaccines/NewsEvents/ WorkshopsMeetingsConferences/ TranscriptsMinutes/UCM054438.pdf

Pollitzer, R. (1954). Plague. WHOMonogr Ser. 22, 1.

Pujol, C., and Bliska, J. B. (2003). The ability to replicate in macrophages is conserved between Yersinia pestis and Yersinia pseudotuberculosis. Infect. Immun. 71, 5892-5899.

Pujol, C., Grabenstein, J. P., Perry, R. D. and Bliska, J. B. (2005). Replication of Yersinia pestis in interferon gammaactivated macrophages requires ripA, a gene encoded in the pigmentation locus. Proc. Natl. Acad. Sci. U.S.A. 102, 12909-12914.

Pujol, C., Klein, K. A., Romanov, G. A., Palmer, L. E., Cirota, C., Zhao, Z., and Bliska, J. B. (2009). Yersinia pestis can reside in autophagosomes and avoid xenophagy in murine macrophages by preventing vacuole acidification. Infect. Immun. 77, 2251-2261.

Qi, Z., Zhou, L., Zhang, Q., Ren, L., Dai, R., Wu, B., Wang, T., Zhu, Z., Yang, Y., Cui, B., Wang, Z., Wang, H., Qiu, Y., Guo, Z., Yang, R., and Wang, X. (2010). Comparison of mouse, guinea pig and rabbit models for evaluation of plague subunit vaccine F1+ rV270. Vaccine $28,1655-1660$. 
Ransom, J. P., and Krueger, A. P. (1954). Chronic pneumonic plague in Macaca mulatta. Am. J. Trop. Med. Hyg. 3, 1040-1054.

Sebbane, F., Gardner, D., Long, D., Gowen, B. B., and Hinnebusch, B. J. (2005). Kinetics of disease progression and host response in a rat model of bubonic plague. Am. J. Pathol. 166, 1427-1439.

Sebbane, F., Lemaitre, N., Sturdevant, D. E., Rebeil, R., Virtaneva, K., Porcella, S. F., and Hinnebusch, B. J. (2006). Adaptive response of Yersinia pestis to extracellular effectors of innate immunity during bubonic plague. Proc. Natl. Acad. Sci. U.S.A. 103, 11766-11771.

Sha, J., Agar, S. L., Baze, W. B., Olano, J. P., Fadl, A. A., Erova, T. E., Wang, S., Foltz, S.M., Suarez, G., Motin, V.L., Chauhan, S., Klimpel, G. R., Peterson, J. W., and Chopra,A.K. (2008). Braun lipoprotein (Lpp) contributes to virulence of yersiniae: potential role of Lpp in inducing bubonic and pneumonic plague. Infect. Immun. 76, 1390-1409.

Sing, A., Roggenkamp, A., Geiger, A. M., and Heesemann, J. (2002a). Yersinia enterocolitica evasion of the host innate immune response by $\mathrm{V}$ antigeninduced IL-10 production of macrophages is abrogated in IL-10-deficient mice. J. Immunol. 168, 1315-1321.

Sing, A., Rost, D., Tvardovskaia, N., Roggenkamp, A., Wiedemann, A., Kirschning, C. J., Aepfelbacher, M., and Heesemann, J. (2002b). Yersinia $\mathrm{V}$-antigen exploits toll-like receptor 2 and CD14 for interleukin 10-mediated immunosuppression. J. Exp. Med. 196, 1017-1024.
Smiley, S. T. (2008a). Current challenges in the development of vaccines for pneumonic plague. Expert Rev. Vaccines 7 , 209-221.

Smiley, S. T. (2008b). Immune defense against pneumonic plague. Immunol. Rev. 225, 256-271.

Speck, R. S., and Wolochow, H. (1957). Studies on the experimental epidemiology of respiratory infections. VIII. Experimental pneumonic plague in Macacus rhesus. J. Infect. Dis. 100, 58-69.

Straley, S. C., and Harmon, P. A. (1984a) Growth in mouse peritoneal macrophages of Yersinia pestis lacking established virulence determinants. Infect. Immun. 45, 649-654.

Straley, S. C., and Harmon, P. A. (1984b). Yersinia pestis grows within phagolysosomes in mouse peritoneal macrophages. Infect. Immun. 45, 655-659.

Strong, R.P., and Teague, O. (1912a).Studies on pneumonic plague and plague immunization. Phillip J. Sci. 7B, 173.

Strong, R. P., and Teague, O. (1912b). Studies on pneumonic plague and plague immunization. VIII. Susceptibility of animals to pneumonic plague. Phillip J. Sci. 7B, 223.

Styer, K. L., Hopkins, G. W., Bartra, S. S., Plano, G. V., Frothingham, R., and Aballay, A. (2005). Yersinia pestis kills Caenorhabditis elegans by a biofilmindependent process that involves novel virulence factors. EMBO Rep. 6, 992-997.

Titball, R. W., and Williamson, E. D. (2001). Vaccination against bubonic and pneumonic plague. Vaccine 19, 4175-4184.
Turner, J. K., McAllister, M. M., Xu, J. L., and Tapping, R. I. (2008). The resistance of $\mathrm{BALB} / \mathrm{cJ}$ mice to Yersinia pestis maps to the major histocompatibility complex of chromosome 17. Infect. Immun. 76, 4092-4099.

Turner, J. K., Xu, J. L., and Tapping, R. I. (2009). Substrains of 129 mice are resistant to Yersinia pestis KIM5: implications for interleukin-10-deficient mice. Infect. Immun. 77, 367-373.

Uddowla, S., Freytag, L. C., and Clements, J. D. (2007). Effect of adjuvants and route of immunizations on the immune response to recombinant plague antigens. Vaccine 25 , 7984-7993.

Une, T., and Brubaker, R. R. (1984). In vivo comparison of avirulent Vwa- and Pgm- or Pstr phenotypes of yersiniae. Infect. Immun. 43, 895-900.

Van Andel, R., Sherwood, R., Gennings, C., Lyons, C. R., Hutt, J., Gigliotti, A., and Barr, E. (2008). Clinical and pathologic features of cynomolgus macaques (Macaca fascicularis) infected with aerosolized Yersinia pestis. Comp. Med. 58, 68-75.

Viboud, G. I., and Bliska, J. B. (2005) Yersinia outer proteins: role in modulation of host cell signaling responses and pathogenesis. Annu. Rev. Microbiol. 59, 69-89.

Warren, J., Walz, U., Reedal, J. S., and Ajl, S. J. (1955). Studies on plague. II. Immunological properties of purified Pasteurella pestis toxin. J. Bacteriol. 70 170-176.

Welkos, S. L., Davis, K. M., Pitt, L. M. Worsham, P. L., and Freidlander, A. M. (1995). Studies on the contribution of the F1 capsule-associated plasmid
pFra to the virulence of Yersinia pestis. Contrib. Microbiol. Immunol. 13 , 299-305.

Williamson, E. D., Flick-Smith, H. C., Lebutt, C., Rowland, C. A., Jones, S. M., Waters, E. L., Gwyther, R. J., Miller, J., Packer, P. J., and Irving, M. (2005). Human immune response to a plague vaccine comprising recombinant $\mathrm{F} 1$ and $\mathrm{V}$ antigens. Infect. Immun. 73, 3598-3608.

Ye,Z., Kerschen, E. J., Cohen, D.A., Kaplan, A. M., van Rooijen, N., and Straley, S. C. (2009). Gr1+ cells control growth of YopM-negative Yersinia pestis during systemic plague. Infect. Immun. 77, 3791-3806.

Conflict of Interest Statement: The author declares that the research was conducted in the absence of any commercial or financial relationships that could be construed as a potential conflict of interest.

Received: 30 August 2010; paper pending published: 13 September 2010; accepted: 11 October 2010; published online: 04 November 2010.

Citation: Lawrenz MB (2010) Model systems to study plague pathogenesis and develop new therapeutics. Front. Microbio. 1:119. doi: 10.3389/fmicb.2010.00119 This article was submitted to Frontiers in Cellular and Infection Microbiology, a specialty of Frontiers in Microbiology. Copyright (c) 2010 Lawrenz. This is an open-access article subject to an exclusive license agreement between the authors and the Frontiers Research Foundation, which permits unrestricted use, distribution, and reproduction in any medium, provided the original authors and source are credited. 